Pure and Applied Mathematics Quarterly

Volume 8, Number 4

$825-834,2012$

\title{
Surfaces Moving by Powers of Gauss Curvature
}

\author{
Ben Andrews and Xuzhong Chen
}

\begin{abstract}
We prove that strictly convex surfaces moving by $K^{\alpha / 2}$ become spherical as they contract to points, provided $\alpha$ lies in the range $[1,2]$. In the process we provide a natural candidate for a curvature pinching quantity for surfaces moving by arbitrary functions of curvature, by finding a quantity conserved by the reaction terms in the evolution of curvature.
\end{abstract}

Keywords: Gauss curvature flow, Curvature estimate.

\section{INTRODUCTION}

In this paper we study the contraction of smooth, compact, convex surfaces with speed given by a power of the Gauss curvature: That is, we consider a family of embeddings $X: S^{2} \times[0, T) \rightarrow \mathbb{R}^{3}$ such that

$$
\frac{\partial X}{\partial t}=-K^{\alpha / 2} \nu
$$

where $\alpha \in[1,2], K$ is the Gauss curvature and $\nu$ denotes the outer unit normal to the evolving surface $M_{t}=X\left(S^{2}, t\right)$.

For $\alpha=2$ this flow coincides with the Gauss curvature flow, which was introduced by Firey [11] as a model for the shape of wearing stones. Firey conjectured that the surfaces should become spherical as they contract to points in this process, and this was confirmed by the first author in [4]. Bennett Chow [10] showed that flow by $K^{\alpha / n}$ with $\alpha>0$ shrinks convex hypersurfaces in $\mathbb{R}^{n+1}$ to points in

Received January 16, 2012

2010 Mathematics Subject Classification. Primary 53C44; Secondary 35K96, 58J35.

Research partly supported by Discovery Projects grants DP0985802 and DP120100097 of the Australian Research Council. The authors are grateful for the hospitality of the Mathematical Sciences Centre of Tsinghua University where this work was carried out. 
finite time, and that the limiting shape is spherical if $\alpha \geq 1$ and the curvature of the initial hypersurface is sufficiently pinched.

Our main theorem is the following:

Theorem 1.1. Let $M_{0}=X_{0}(M)$ be a compact, smooth, strictly convex surface in $\mathbb{R}^{3}$, given by an embedding $X_{0}$. Then there exists a maximal $T>0$ and a unique, smooth solution $\left\{M_{t}=X_{t}(M)\right\}$ of the evolution equation (1.1) with $M_{0}=X_{0}(M)$. The surfaces $M_{t}$ are smooth and strictly convex, and converge to $q \in \mathbb{R}^{3}$ as $t$ approaches $T$. Rescaling about $q$ gives smooth convergence to a sphere

$$
\tilde{X}(t)=\frac{X-q}{((\alpha+1)(T-t))^{\frac{1}{\alpha+1}}} \rightarrow \tilde{X}_{T}
$$

in $C^{\infty}$, where $\tilde{X}_{T}$ is a smooth embedding with $\tilde{X}_{T}(M)=\mathbb{S}^{2}(1) \subset \mathbb{R}^{3}$.

The existence and uniqueness, smoothness and convexity of the solutions, and the uniform convergence to a point, were all proved in [10]. Our contribution is the last part of the above theorem. The key step in the proof is a pinching estimate for the principal curvatures, analogous to that used in [4]. We remark that the evolution of surfaces by curvature is rather well understood in the case where the speed is homogeneous of degree one in the principal curvatures [8], but far less is known for other degrees of homogeneity: There are a few flows where curvature estimates have been found which are strong enough to deduce that limiting shapes are spheres, starting with the Gauss curvature flow in [4]. A collection of other flows have been treated [13,14], including certain powers of mean curvature and certain sums of powers of principal curvatures, for which curvature pinching estimates were found using a computational search algorithm. However at present we are far from understanding such flows of surfaces in any generality. One of the contributions of the present paper is a natural candidate for a quantity which measures the pinching of principal curvatures to each other as the surface shrinks to a point: We solve the system of ordinary differential equations associated with the 'reaction' terms in the evolution of curvature for quite general flows by functions of curvature. We hope this method may be useful for a wider class of flows, and mention at the end of the paper several other classes of flows for which this quantity is preserved. 


\section{PROOF OF THE THEOREM}

We work with a description of uniformly convex surfaces using their support function as in [2]: For each $z \in S^{2} \subset \mathbb{R}^{3}$ we define $s(z, t)=\sup \left\{x \cdot z: x \in M_{t}\right\}$. Then the surface $M_{t}$ is recovered by the embedding $X(z, t)=s(z, t) z+\nabla s(z, t)$, where $\nabla s$ is the gradient vector of $s(., t)$ on $S^{2}$, interpreted as a vector in $\mathbb{R}^{3}$ tangent to $S^{2}$ at $z$. The principal radii of curvature (denoted $r_{1}$ and $r_{2}$ ) are the eigenvalues of the symmetric positive definite bilinear form $\mathfrak{r}_{i j}=\nabla_{i} \nabla_{j} s+g_{i j} s$, where $g_{i j}$ is the standard metric on $S^{2}$, and $\nabla$ the corresponding connection. The evolution equation (1.1) is then equivalent to the scalar parabolic equation

$$
\frac{\partial s}{\partial t}=F\left(\mathfrak{r}_{i j}\right),
$$

where $F(A)=-\operatorname{det}(A)^{-\alpha / 2}$. The Codazzi identity implies that $\nabla_{i} \mathfrak{r}_{j k}$ is totally symmetric. The following evolution equation holds for $\mathfrak{r}_{i j}$ (see for example $[5$, Equation 20]):

$$
\frac{\partial}{\partial t} \mathfrak{r}_{i j}=\dot{F}^{k l} \nabla_{k} \nabla_{l} \mathfrak{r}_{i j}+\ddot{F}^{k l, m n} \nabla_{i} \mathfrak{r}_{k l} \nabla_{j} \mathfrak{r}_{m n}+\left(F+\dot{F}^{k l} \mathfrak{r}_{k l}\right) g_{i j}-\dot{F}^{k l} g_{k l} \mathfrak{r}_{i j},
$$

where $\dot{F}^{i j}=\frac{\alpha}{2} \operatorname{det}(A)^{-\alpha / 2}\left(\mathfrak{r}^{-1}\right)^{i j}$ is the matrix of derivatives of $F$ with respect to the components of $\mathfrak{r}_{i j}$, and $\ddot{F}$ gives the corresponding second derivatives.

2.1. The pinching estimate. In this section we prove an pinching estimate of curvature and convergence of the evolving surfaces. We begin with the following lemma.

Lemma 2.1. If $\mathfrak{r}_{i j} \leq C g_{i j}$ at $t=0$, then this remains true for all $t \in[0, T)$.

Proof. Consider $M_{i j}=\mathfrak{r}_{i j}-C g_{i j}$ with $C>0$ so large that $M_{i j} \leq 0$ at $t=0$. We wish to show that $M_{i j} \leq 0$ for $t \in[0, T)$. By $(2.2)$, we have:

$$
\frac{\partial}{\partial t} M_{i j}=\dot{F}^{k l} \nabla_{k} \nabla_{l} M_{i j}+\ddot{F}^{k l, m n} \nabla_{i} M_{k l} \nabla_{j} M_{m n}+(1-\alpha) F g_{i j}-\dot{F}^{k l} g_{k l} \mathfrak{r}_{i j},
$$

Let $v$ be a zero eigenvector of $M_{i j}$ with $|v|=1, M_{i j} v^{j}=\left(\mathfrak{r}_{i j}-C g_{i j}\right) v^{j}=0$. Then we have

$$
\left((1-\alpha) F g_{i j}-\dot{F}^{k l} g_{k l} \mathfrak{r}_{i j}\right) v^{i} v^{j}=F\left((1-\alpha) g_{i j}+\alpha g_{i j}\right) v^{i} v^{j}=F<0
$$

since $F$ is a concave function of the components $\mathfrak{r}_{i j}$. The result follows from Hamilton's maximum principle [12, Theorem 9.1]. 
Theorem 2.2. Let $\left\{M_{t}=X(M, t)_{0 \leq t<T}\right\}$ be a smooth, strictly convex solution of the flow equation (1.1). Then

$$
\sup _{M} \frac{\left(r_{1}(x, t)-r_{2}(x, t)\right)^{2}}{\left(r_{1}(x, t) r_{2}(x, t)\right)^{\alpha}} \leq \sup _{M} \frac{\left(r_{1}(x, 0)-r_{2}(x, 0)\right)^{2}}{\left(r_{1}(x, 0) r_{2}(x, 0)\right)^{\alpha}}
$$

Proof. We order the principal radii so that $r_{2}>r_{1}$. Then $r_{2}$ and $r_{1}$ may not be smooth functions on $M$, but they are smooth on a neighbourhood of any non-umbillic point. For small $\varepsilon>0$ we will prove the negativity of the quantity

$$
G=-F\left(r_{2}-r_{1}\right)-C_{t}
$$

where $C_{t}=C_{0}+\varepsilon(1+t)$ and $C_{0}=\sup _{M} \frac{r_{2}(x, 0)-r_{1}(x, 0)}{\left(r_{1}(x, 0) r_{2}(x, 0)\right)^{\alpha / 2}}$. This choice makes $G$ strictly negative for $t=0$. Note that $G$ is smooth near any point where $G=0$, since such a point cannot be umbillic. If $G$ is not everywhere negative, we consider the first time $t$ where the maximum of $G$ reaches zero, and a point $p$ where this occurs. Using (2.2) we obtain the following evolution equation for $G$ :

$$
\begin{aligned}
\frac{\partial G}{\partial t} & =\dot{F}^{i j} \nabla_{i} \nabla_{j} G+\left(\dot{G}^{i j} \ddot{F}^{k l, m n}-\dot{F}^{i j} \ddot{G}^{k l, m n}\right) \nabla_{i} \mathfrak{r}_{k l} \nabla_{j} \mathfrak{r}_{m n}, \\
& +\left(F+\dot{F}^{i j} \mathfrak{r}_{i j}\right) \dot{G}^{i j} g_{i j}-\dot{F}^{k l} g_{k l} \dot{G}^{i j} \mathfrak{r}_{i j}-\varepsilon .
\end{aligned}
$$

Choose local coordinates for $M$ near $p$ such that $\mathfrak{r}_{i j}(p, t)=\operatorname{diag}\left(r_{1}, r_{2}\right)$. For convenience we use the notation $\dot{f}^{i}=\frac{\partial F}{\partial r_{i}}$ and $\ddot{f}^{i j}=\frac{\partial^{2} F}{\partial r_{i} \partial r_{j}}$, and similarly $\dot{g}^{i}=\frac{\partial G}{\partial r_{i}}$ and $\ddot{g}^{i j}=\frac{\partial^{2} G}{\partial r_{i} \partial r_{j}}$. We now estimate the second term on the right hand side of the above evolution equation. To do this we use the method of [6] to reduce the negativity of this term to a pair of inequalities. The computation uses results from [7] which give the first and second derivatives of $F$ and $G$ in the above orthonormal frame:

$$
\begin{gathered}
\ddot{F}^{11,11}=\ddot{f}^{11}, \quad \ddot{F}^{22,22}=\ddot{f}^{22}, \quad \ddot{F}^{11,22}=\ddot{f}^{12}, \\
\ddot{F}^{12,12}=\ddot{F}^{21,21}=\frac{\dot{f}^{1}-\dot{f}^{2}}{r_{1}-r_{2}}
\end{gathered}
$$


It follows that the second term on the right hand side of (2.4) is as follows:

$$
\begin{aligned}
Q & :=\left(\dot{G}^{i j} \ddot{F}^{k l, m n}-\dot{F}^{i j} \ddot{G}^{k l, m n}\right) \nabla_{i} \mathfrak{r}_{k l} \nabla_{j} \mathfrak{r}_{m n} \\
& =\left(\dot{g}^{1} \ddot{f}^{11}-\dot{f}^{1} \ddot{g}^{11}\right)\left(\nabla_{1} \mathfrak{r}_{11}\right)^{2}+\left(\dot{g}^{1} \ddot{f}^{22}-\dot{f}^{1} \ddot{g}^{22}\right)\left(\nabla_{1} \mathfrak{r}_{22}\right)^{2} \\
& +2\left(\dot{g}^{1} \ddot{f}^{12}-\dot{f}^{1} \ddot{g}^{12}\right) \nabla_{1} \mathfrak{r}_{11} \nabla_{1} \mathfrak{r}_{22}+2 \frac{\dot{g}^{1} \dot{f}^{2}-\dot{f}^{1} \dot{g}^{2}}{r_{2}-r_{1}}\left(\nabla_{1} \mathfrak{r}_{12}\right)^{2} \\
& +\left(\dot{g}^{2} \ddot{f}^{11}-\dot{f}^{2} \ddot{g}^{11}\right)\left(\nabla_{2} \mathfrak{r}_{11}\right)^{2}+\left(\dot{g}^{2} \ddot{f}^{22}-\dot{f}^{2} \ddot{g}^{22}\right)\left(\nabla_{2} \mathfrak{r}_{22}\right)^{2} \\
& +2\left(\dot{g}^{2} \ddot{f}^{12}-\dot{f}^{2} \ddot{g}^{12}\right) \nabla_{2} \mathfrak{r}_{11} \nabla_{2} \mathfrak{r}_{22}+2 \frac{\dot{g}^{1} \dot{f}^{2}-\dot{g}^{2} \dot{f}^{1}}{r_{2}-r_{1}}\left(\nabla_{2} \mathfrak{r}_{12}\right)^{2}
\end{aligned}
$$

At the maximum point $(p, t)$ of $G$, we have $G=0$ and $\nabla_{i} G=0$. The latter gives two equations which may be written as follows:

$$
T_{1}:=\frac{\nabla_{1} \mathfrak{r}_{11}}{\dot{g}^{2}}=-\frac{\nabla_{1} \mathfrak{r}_{22}}{\dot{g}^{1}} ; \quad T_{2}:=\frac{\nabla_{2} \mathfrak{r}_{22}}{\dot{g}^{1}}=-\frac{\nabla_{2} \mathfrak{r}_{11}}{\dot{g}^{2}} .
$$

These identities and the Codazzi symmetries $\nabla_{1} \mathfrak{r}_{12}=\nabla_{2} \mathfrak{r}_{11}$ and $\nabla_{2} \mathfrak{r}_{12}=\nabla_{1} \mathfrak{r}_{22}$ reduce $Q$ to a linear combination of $T_{1}^{2}$ and $T_{2}^{2}$ : We have $Q=Q_{1} T_{1}^{2}+Q_{2} T_{2}^{2}$, where

$$
\begin{aligned}
& Q_{1}=\left(\dot{g}^{1} \ddot{f}-\dot{f}^{1} \ddot{g}\right)\left(\dot{g}^{2} e_{1}-\dot{g}^{1} e_{2}, \dot{g}^{2} e_{1}-\dot{g}^{1} e_{2}\right)+2\left(\dot{g}^{1}\right)^{2} \frac{\dot{g}^{1} \dot{f}^{2}-\dot{g}^{2} \dot{f}^{1}}{r_{2}-r_{1}} \\
& Q_{2}=\left(\dot{g}^{2} \ddot{f}-\dot{f}^{2} \ddot{g}\right)\left(\dot{g}^{2} e_{1}-\dot{g}^{1} e_{2}, \dot{g}^{2} e_{1}-\dot{g}^{1} e_{2}\right)+2\left(\dot{g}^{2}\right)^{2} \frac{\dot{g}^{1} \dot{f}^{2}-\dot{g}^{2} \dot{f}^{1}}{r_{2}-r_{1}} .
\end{aligned}
$$

Now using the expression $g=-f\left(r_{2}-r_{1}\right)-C_{t}$ we obtain

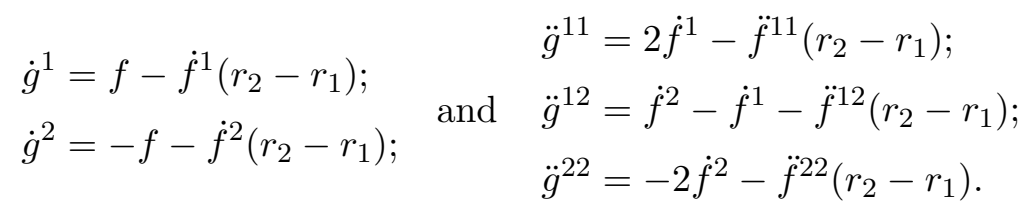

The terms involving $\ddot{f}$ produce significant cancellations, resulting in the following:

$$
\begin{aligned}
Q_{1}= & f \ddot{f}\left(\dot{g}^{2} e_{1}-\dot{g}^{1} e_{2}, \dot{g}^{2} e_{1}-\dot{g}^{1} e_{2}\right) \\
& +2 f\left(\dot{f}^{1}+\dot{f}^{2}\right)\left(\frac{f^{2}}{r_{2}-r_{1}}-2 f \dot{f}^{1}-\dot{f}^{1} \dot{f}^{2}\left(r_{2}-r_{1}\right)\right) ; \\
Q_{2}= & -f \ddot{f}\left(\dot{g}^{2} e_{1}-\dot{g}^{1} e_{2}, \dot{g}^{2} e_{1}-\dot{g}^{1} e_{2}\right) \\
& +2 f\left(\dot{f}^{1}+\dot{f}^{2}\right)\left(\frac{f^{2}}{r_{2}-r_{1}}+2 f \dot{f}^{2}-\dot{f}^{1} \dot{f}^{2}\left(r_{2}-r_{1}\right)\right) .
\end{aligned}
$$


Now we use the homogeneity of the speed $f$ : Write $f=-k^{-\alpha}$, where $k$ is homogeneous of degree one. This gives

$$
\dot{f}:=\alpha k^{-(1+\alpha)} \dot{k} ; \quad \text { and } \quad \ddot{f}:=-\alpha(1+\alpha) k^{-(2+\alpha)} \dot{k} \otimes \dot{k}+\alpha k^{-(1+\alpha)} \ddot{k} .
$$

The term $\ddot{f}\left(\dot{g}^{2} e_{1}-\dot{g}^{1} e_{2}, \dot{g}^{2} e_{1}-\dot{g}^{1} e_{2}\right)$ in (2.6) and (2.7) appears to involve fourth powers of $\alpha$, with two powers from $\ddot{k}$ and one from each factor $\dot{g}^{2} e_{1}-\dot{g}^{1} e_{2}$. However, these are proportional to $(\dot{k} \otimes \dot{k})\left(\dot{k}^{2} e_{1}-\dot{k}^{1} e_{2}, \dot{k}^{2} e_{1}-\dot{k}^{1} e_{2}\right)=\left(\dot{k}\left(\dot{k}^{2} e_{1}-\right.\right.$ $\left.\dot{k}^{1} e_{2}\right)^{2}$, which is zero. Thus this term involves at most three powers of $\alpha$. Since there is a common factor $\alpha$, this term is equal to $\alpha$ multiplied by a quadratic function of $\alpha$. The other terms in $Q_{1}$ and $Q_{2}$ are of degree at most three in $\alpha$ and have a factor $\alpha$, so we have the following:

Lemma 2.3. $\frac{Q_{1}}{\alpha}$ and $\frac{Q_{2}}{\alpha}$ are quadratic functions of $\alpha$.

We now show the following:

Lemma 2.4. $\frac{Q_{1}}{\alpha}$ and $\frac{Q_{2}}{\alpha}$ are convex functions of $\alpha$.

Proof. By Lemma 2.3 we must show the $\alpha^{3}$ terms in $Q_{1}$ and $Q_{2}$ are non-negative. In the first line of $(2.6),-\alpha^{3} k^{-3-4 \alpha}\left(r_{2}-r_{1}\right)^{2} \ddot{k}\left(\dot{k}^{2} e_{1}-\dot{k}^{1} e_{2}, \dot{k}^{2} e_{1}-\dot{k}^{1} e_{2}\right)$ is the only such term. From the second line of (2.6) only the last term in the bracket contributes, yielding $2 \alpha^{3} k^{-3-4 \alpha}\left(r_{2}-r_{1}\right) \dot{k}^{1} \dot{k}^{2}\left(\dot{k}^{1}+\dot{k}^{2}\right)$. In the expression (2.7) for $Q_{2}$ the same two terms arise, but the sign of the first is reversed. Thus the condition for both $Q_{2} / \alpha$ and $Q_{1} / \alpha$ to be convex is precisely

$$
2 \dot{k}^{1} \dot{k}^{2}\left(\dot{k}^{1}+\dot{k}^{2}\right) \geq\left(r_{2}-r_{1}\right)\left|\ddot{k}\left(\dot{k}^{2} e_{1}-\dot{k}^{1} e_{2}, \dot{k}^{2} e_{1}-\dot{k}^{1} e_{2}\right)\right| .
$$

For Gauss curvature flows $k=\sqrt{r_{1} r_{2}}$, so the left hand side is $\frac{1}{4}\left(\sqrt{\frac{r_{2}}{r_{1}}}+\sqrt{\frac{r_{1}}{r_{2}}}\right)$, while the right is $\frac{1}{4}\left|\sqrt{\frac{r_{2}}{r_{1}}}-\sqrt{\frac{r_{1}}{r_{2}}}\right|$. The inequality clearly holds.

It was proved in [4] that $Q_{1}$ and $Q_{2}$ are non-positive when $\alpha=2$, and in [8] that $Q_{1}$ and $Q_{2}$ are non-positive for $\alpha=1$ (for any $k$ ). By Lemma 2.4, $Q_{1}$ and $Q_{2}$ are non-positive for any $\alpha \in[1,2]$, and hence $Q \leq 0$. 
Now we consider the first two terms on the second line of (2.4):

$$
\begin{aligned}
Z:= & \left(F+\dot{F}^{i j} \mathfrak{r}_{i j}\right) \dot{G}^{i j} g_{i j}-\dot{F}^{k l} g_{k l} \dot{G}^{i j} \mathfrak{r}_{i j} \\
= & \left(f+\dot{f}^{1} r_{1}+\dot{f}^{2} r_{2}\right)\left(\dot{g}^{1}+\dot{g}^{2}\right)-\left(\dot{f}^{1}+\dot{f}^{2}\right)\left(\dot{g}^{1} r_{1}+\dot{g}^{2} r_{2}\right) \\
= & \left(f+\dot{f}^{1} r_{1}+\dot{f}^{2} r_{2}\right)\left(-\dot{f}^{1}\left(r_{2}-r_{1}\right)+f-\dot{f}^{2}\left(r_{2}-r_{1}\right)-f\right) \\
& -\left(\dot{f}^{1}+\dot{f}^{2}\right)\left(\left(-\dot{f}^{1}\left(r_{2}-r_{1}\right)+f\right) r_{1}+\left(-\dot{f}^{2}\left(r_{2}-r_{1}\right)-f\right) r_{2}\right) \\
= & -\left(f+\dot{f}^{1} r_{1}+\dot{f}^{2} r_{2}\right)\left(\dot{f}^{1}+\dot{f}^{2}\right)\left(r_{2}-r_{1}\right) \\
& -\left(\dot{f}^{1}+\dot{f}^{2}\right)\left(-f-\dot{f}^{1} r_{1}-\dot{f}^{2} r_{2}\right)\left(r_{2}-r_{1}\right) \\
= & 0 .
\end{aligned}
$$

Since the last term on the second line of (2.4) is strictly negative, we arrive at a contradiction. Therefore $G$ remains negative as long as the solution exists, and allowing $\varepsilon$ to approach zero proves the Theorem.

Corollary 2.5. For a smooth compact strictly convex surface $M_{t}$ in $\mathbb{R}^{3}$, flowing according to $\frac{\partial X}{\partial t}=-K^{\alpha / 2} \nu$ with $1 \leq \alpha \leq 2$, there exists $C_{1}=C_{1}\left(M_{0}, \alpha\right)$ such that $0<\frac{1}{C_{1}} \leq \frac{r_{2}}{r_{1}} \leq C_{1}$.

Proof. Choose $C>0$ such that $r_{1}, r_{2}<C$ at $t=0$. Lemma 2.1 and Theorem 2.2 implies that

$$
C^{2-2 \alpha}\left(\frac{r_{1}}{r_{2}}+\frac{r_{2}}{r_{1}}-2\right) \leq\left(r_{1} r_{2}\right)^{1-\alpha} \frac{\left(r_{1}-r_{2}\right)^{2}}{r_{1} r_{2}}=\frac{\left(r_{1}-r_{2}\right)^{2}}{\left(r_{1} r_{2}\right)^{\alpha}} \leq C_{0}^{2} .
$$

We obtain the bound on $\frac{r_{2}}{r_{1}}$ claimed above.

2.2. Convergence. Given the pinching estimate of Theorem 2.2, the proof of convergence is rather straightforward: We already know that the surfaces remain smooth until they contract to a point, from the result of [10]. From Theorem 2.2 we can deduce a strong pinching result of the kind used in [9] as follows: Ordering the principal radii as $r_{2} \geq r_{1}$ as before, the principal curvatures $\kappa_{i}=1 / r_{i}$ satisfy $\kappa_{1} \geq \kappa_{2}$, and Theorem 2.2 implies that

$$
\kappa_{1}-\kappa_{2} \leq C_{0}\left(\kappa_{1} \kappa_{2}\right)^{\frac{2-\alpha}{2}} .
$$

By corollary 2.5 we have

$$
\kappa_{1}-\kappa_{2} \leq C_{0} C_{1}^{\frac{2-\alpha}{2}} \kappa_{2}^{2-\alpha}
$$


For $\alpha \in(1,2)$ we have $2-\alpha \in(0,1)$. By Young's inequality we have for any $\varepsilon>0$

$$
\kappa_{2}^{2-\alpha} \leq \varepsilon \kappa_{2}+(\alpha-1)(2-\alpha)^{\frac{2-\alpha}{\alpha-1}} \varepsilon^{-\frac{2-\alpha}{1-\alpha}} .
$$

Thus it follows that for any $\varepsilon>0$ there exists $C(\varepsilon)$ such that

$$
\kappa_{1} \leq(1+\varepsilon) \kappa_{2}+C(\varepsilon)
$$

The argument of [9, Theorem 3.1] applies to prove that for any $\delta>0$ there exists $r(\delta)>0$ such that the ratio of circumradius $r_{+}\left(M_{t}\right)$ to inradius $r_{-}\left(M_{t}\right)$ is bounded by $1+\delta$ provided $r_{+}(M)<r(\delta)$. Then [9, Proposition 12.1] applies to prove that the speed $K^{\alpha / 2}$ is bounded below by $C(T-t)^{-\frac{\alpha}{1+\alpha}}$ for $t$ sufficiently close to $T$, and the higher regularity and convergence of the rescaled hypersurfaces follow as described in [9, Section 13].

\section{REMARKS AND EXTENSIONS}

Remark 3.1. In the proof of the pinching estimate we used the structure of the Gauss curvature flows only in a few places: In the proof that the zero order terms $Z$ in equation (2.4) vanish we did not use any information about the speed, so this part of the argument works for arbitrary flows of surfaces by curvature, and amounts to a closed form solution of the 'reaction' part of the evolution of curvature in such flows. In the proof of non-positivity of the gradient terms $Q$ we used the homogeneity of the speed, but only the particular speed $K^{\alpha / 2}$ at the last stage. We expect this argument will apply for a reasonably large family of flows with high degree of homogeneity $\alpha$, but emphasise that the argument relied on the fact that the case $\alpha=2$ is already known from [4] (the case $\alpha=1$ is known for very general flows from [8]).

Remark 3.2. Although we used only the highest order terms in $\alpha$ in the proof, one can of course compute $Q_{1}$ and $Q_{2}$ completely for flows by powers of Gauss curvature. This is a somewhat lengthy but straightforward computation, and yields

$$
\begin{aligned}
& \frac{Q_{1}}{2 \alpha}=\frac{\left(2 \alpha^{2}-5 \alpha+2\right) r_{1}^{2} r_{2}^{3}-\left(4 \alpha^{2}-7 \alpha+6\right) r_{1}^{3} r_{2}^{2}+\left(2 \alpha^{2}-3 \alpha-2\right) r_{1}^{4} r_{2}+(\alpha-2) r_{1}^{5}}{\left(r_{1} r_{2}\right)^{\alpha / 2+2}\left(r_{2}-r_{1}\right)\left(\alpha r_{2}+(2-\alpha) r_{1}\right)^{2}} \\
& \frac{Q_{2}}{2 \alpha}=\frac{\left(2 \alpha^{2}-5 \alpha+2\right) r_{2}^{2} r_{1}^{3}-\left(4 \alpha^{2}-7 \alpha+6\right) r_{2}^{3} r_{1}^{2}+\left(2 \alpha^{2}-3 \alpha-2\right) r_{2}^{4} r_{1}+(\alpha-2) r_{2}^{5}}{\left(r_{1} r_{2}\right)^{\alpha / 2+2}\left(r_{2}-r_{1}\right)\left(\alpha r_{1}+(2-\alpha) r_{2}\right)^{2}}
\end{aligned}
$$


One can then check directly that $Q_{1}$ and $Q_{2}$ are non-positive for $\alpha$ in the range $[1,2]$. We can also see from these expressions that the pinching estimate fails for $\alpha>2$ if $r_{2} / r_{1}$ is large, since the coefficient of $r_{2}^{5}$ in the numerator of $Q_{2}$ is then positive. One can also check that $Q_{1}$ and $Q_{2}$ are non-positive for $\alpha=1 / 2$, and hence by the above argument for $\alpha \in[1 / 2,2]$, but not for $0<\alpha<\frac{1}{2}$. The resulting pinching estimate for $\alpha<1$ is not useful for surfaces in Euclidean space, but is a strong pinching estimate for convex spacelike co-compact hypersurfaces in Minkowski space $\mathbb{R}^{2,1}$. The flow for $\alpha=1 / 2$ is particularly interesting because it is the affine invariant flow studied for Euclidean convex hypersurfaces in [3].

Remark 3.3. The solution of the 'reaction' ODE system in the form $F\left(\mathfrak{r}_{2}-\mathfrak{r}_{1}\right)=c$ provides a natural choice of pinching quantity to consider for other flows. The conditions required on $F$ to make the gradient terms favourable to preserve this condition are not simple to understand in any generality, but can be checked in concrete examples: We announce here some results of this investigation, details of which will be provided in a separate paper: Applying this recipe for flows by powers $H^{\alpha}$ of the mean curvature we find the quantity $\sup _{M_{t}} H^{\alpha}\left|\kappa_{2}-\kappa_{1}\right| / K$ is non-increasing under the flow provided $1 \leq \alpha \leq \alpha_{*}$ where $\alpha_{*}$ is approximately 5.16. These flows were considered previously in [13], where pinching estimates were found for $\alpha=2,3,4$, and in [14] where pinching quantities were found for $1 \leq \alpha \leq 5$. Similarly we can consider flow by powers of $|A|=\sqrt{\kappa_{1}^{2}+\kappa_{2}^{2}}$ : Here our pinching estimate $|A|^{\alpha}\left|\kappa_{2}-\kappa_{1}\right| / K \leq C$ applies for $\alpha \geq 1$ up to approximately 8.15 (the values $\alpha=2$ and $\alpha=4$ were considered in [13]). Finally, in the case where $F=\kappa_{1}^{\alpha}+\kappa_{2}^{\alpha}$, the pinching estimate $F\left|\kappa_{2}-\kappa_{1}\right| / K \leq C$ holds for any $\alpha>1$. This is the first example where flows of arbitrarily high degree of homogeneity have been shown to flow convex surfaces to spheres.

\section{REFERENCES}

[1] Ben Andrews, Contraction of convex hypersurfaces in Euclidean space, Calc. Var. Partial Differential Equations 2 (1994), no. 2, 151-171.

[2] — Harnack inequalities for evolving hypersurfaces, Math. Z. 217 (1994), no. 2, 179197.

[3] - Contraction of convex hypersurfaces by their affine normal, J. Differential Geom. 43 (1996), no. 2, 207-230.

[4] Gauss curvature flow: the fate of the rolling stones, Invent. Math. 138 (1999), no. $1,151-161$. 
[5] Motion of hypersurfaces by Gauss curvature, Pacific J. Math. 195 (2000), no. 1, $1-34$.

[6] - Positively curved surfaces in the three-sphere, (Beijing, 2002), Higher Ed. Press, Beijing, 2002, pp. 221-230.

[7] _ Pinching estimates and motion of hypersurfaces by curvature functions, J. Reine Angew. Math. 608 (2007), 17-33.

[8] Moving surfaces by non-concave curvature functions, Calc. Var. Partial Differential Equations 39 (2010), no. 3-4, 649-657.

[9] Ben Andrews and James A. McCoy, Convex Hypersurfaces with pinched curvatures and flow of convex hypersurfaces by high powers of curvature, Trans. Amer. Math. Soc. 364 (2012), no. 7, 3427-3447.

[10] Bennett Chow, Deforming convex hypersurfaces by the nth root of the Gaussian curvature, J. Differential Geom. 22 (1985), no. 1, 117-138.

[11] William J. Firey, Shapes of worn stones, Mathematika 21 (1974), 1-11.

[12] Richard S. Hamilton, Three-manifolds with positive Ricci curvature, J. Differential Geom. 17 (1982), no. 2, 255-306.

[13] Oliver C. Schnürer, Surfaces contracting with speed $|A|^{2}$, J. Differential Geom. 71 (2005), no. 3, 347-363.

[14] Felix Schulze, Convexity estimates for flows by powers of the mean curvature, Ann. Sc. Norm. Super. Pisa Cl. Sci. (5) 5 (2006), no. 2, 261-277.

[15] Kaising Tso, Deforming a hypersurface by its Gauss-Kronecker curvature, Comm. Pure Appl. Math. 38 (1985), no. 6, 867-882.

Ben Andrews

Mathematical Sciences Center, Tsinghua University;

Mathematical Sciences Institute, Australia National University;

and Morningside Center for Mathematics, Chinese Academy of Sciences.

E-mail: Ben.Andrews@anu.edu.au

Xuzhong Chen

Mathematical Sciences Center

Tsinghua University

E-mail: cxzmath@yahoo.com.cn 